\title{
TIMBRE-BASED COMPOSITION,
} MULTIPLE PERSPECTIVES AND

\section{AMBIGUITY IN REBECCA SAUNDERS' COMPOSITIONAL STYLE}

\author{
Omri Abram
}

\begin{abstract}
This article takes up Rebecca Saunders' comments on the significance of timbre in her work to develop a timbre-centred analytical technique for two of her compositions, Ire (2012), for cello and ensemble, and Still (2011), for violin and orchestra. Two overarching principles are identified: the organisation of the pieces' sounds into clearly differentiated categories, which can also overlap in different ways, and the use of a phrase-based logic in the pieces' formal construction. Alongside the timbral construction, stable pitches acquire formal significance by virtue of their rarity and conspicuousness. I also elaborate on the ways in which perceptual ambiguity is fruitfully exploited in these works.
\end{abstract}

\section{Introduction}

When I started composing I was passionately interested in the timbral mutating of one sound into another. It wasn't of course a conscious decision, but something which felt natural and fascinated me.

(Rebecca Saunders, interview with the author ${ }^{1}$ )

Rebecca Saunders (b. 1967) has often mentioned timbre as a main focus of her compositions. This article presents a timbre-based analysis of two works by Saunders: Ire, for cello and ensemble (2012), and Still, for violin and orchestra (2011). Timbre-based analyses of contemporary works are rare, for several reasons. ${ }^{2}$ In contrast to pitch and rhythm, timbre encompasses a vast field of musical phenomena, making it more difficult to define empirically. Consequently, scholarly examination of musical timbre is generally confined to the field of psychoacoustics, where the complex multidimensional interplay of timbre's acoustic components is diminished in the design of experiments that often target a specific attribute. Since most aspects of timbre cannot be assigned absolute values (as in pitch), the empirical results often lack ecological validity in listening to complex real-world sounds or actual musical pieces. ${ }^{3}$ As a result, timbre-based analysis of

\footnotetext{
1 Rebecca Saunders, email interview, 14 April 2017.

2 For one such example, albeit with a markedly different analytical approach to the one used in this article, see Danuta Mirka, 'To Cut the Gordian Knot: The Timbre System of Krzysztof Penderecki', Journal of Music Theory, 45, no. 2 (2001), pp. 435-56.
} 
music relies to some extent on the subjective perception of pieces' aural building blocks. ${ }^{4}$

The dearth of timbre-based analyses of twentieth- and twenty-first-century music, is, however, somewhat surprising. The growing significance of timbre in musical composition over the past century, and the many works in which timbre is arguably a central facet, would seem to render traditional pitch- and rhythm-based analyses inadequate for their examination. I hope to demonstrate through the two examined pieces the validity of a timbre-centred approach, particularly in dealing with the use of perceptual ambiguity, which plays a central role in both works. By perceptual ambiguity I refer to aural contexts that foster multiple possible perceptual groupings and interpretations of the same sounds.

\section{Classification of sound materials in Ire: a timbre-based approach}

The majority of sound materials in Ire avoid stable and sustained pitches through extensive use of glissandi, trills, tremolos, changes in bow position or exaggerated vibrato in the string instruments, alongside the use of percussion instruments with complex and rapidly changing timbres. This lends timbral aspects of the musical surface a primary significance; consequently, the sound materials can be better classified through qualities such as brightness, shrillness, dullness, types of attack or decay and register, rather than pitch. Despite the general instability of the sound materials in Ire, I find that they can be sorted into clearly differentiated and easily recognisable timbral categories. Some of the categories are defined by one main timbral attribute (such as range), while others result from the combination of a relatively small number of components. Additionally, the categories may sometimes overlap, revealing a middle ground between them. My analysis focuses on the structure of the piece as a process of differentiation and/or morphing between timbral categories.

\section{A Proposed List of Sound Categories in Ire}

The first two categories I will describe were so designated by Saunders. In her preface to the piece, ${ }^{5}$ she describes 'two Elemental Sounds' that are explored during the piece, with 'a gradual process of fusion' between them:

(1) First 'Elemental Sound' (Element 1). This is described by Saunders as 'solo violoncello: initial low timbral palette explores... the rich sonority of the fourth string when scordatura down one octave' (see Example 1). This category is fairly broad and encompasses many sounds, defined primarily by use of the low register, while modulating the timbre through changes in dynamics, vibrato, glissandi, trills, etc. This type of sound appears

\footnotetext{
${ }^{3}$ For an exemplary overview of psychoacoustic research into timbre, see Stephen McAdams, 'Perspectives on the Contribution of Timbre to Musical Structure', Computer Music Journal 23, no. 3 (1999), pp. 85-102.

${ }^{4}$ In this context, it is worth mentioning the attempts at classifying complex sounds using a phenomenological approach - most importantly, those of Pierre Schaeffer in his Treatise on Musical Objects. Pierre Schaeffer, Treatise on Musical Objects: An Essay across Disciplines, translated by John Dack and Christine North, (Oakland: University of California Press, 2017; French-language original, 1966). For an overview, see Omri Abram, 'Sound, Form and Meaning: Pierre Schaeffer and Helmut Lachenmann - a Comparison', Master's thesis (Hebrew University of Jerusalem, 2019).

5 Rebecca Saunders, Ire (Leipzig: C. F. Peters, 2012).
} 


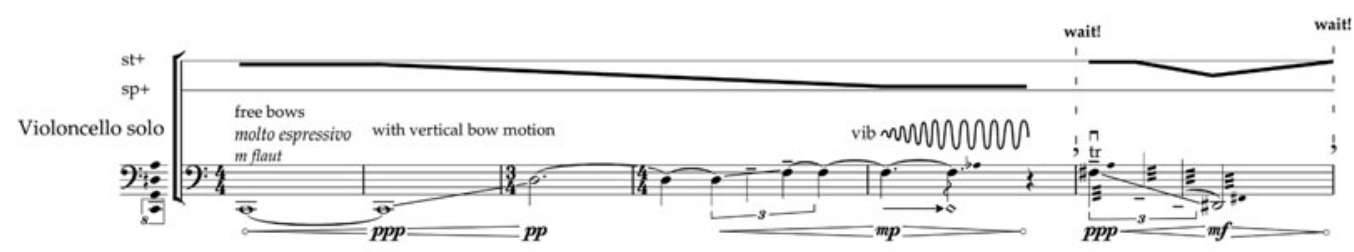

Example 1.

Rebecca Saunders, Ire, solo cello part, bars 1-6. (C) Copyright 2016 Henry Litolff's Verlag. mainly in the soloist's part (whose C string is tuned one octave lower), serving to differentiate the soloist from the ensemble. However, some sounds produced by the ensemble may be included in this same category, especially in the double bass (itself with a scordatura lowering its range), ensemble cello and percussion (mainly actions on the bass drum and tam-tam). Because of the low range, precise pitch becomes harder to distinguish ${ }^{6}$ and is therefore of relatively marginal salience in the perception of the sound. Moreover, the pitch is sometimes altered by changes in bow pressure and/or dynamics (as in bar 7).

(2) Second 'Elemental Sound' (Element 2). According to Saunders, this is 'in its purest form, an up-bow double harmonic trill moving towards sul ponticello, with a fast and smooth glissando'. This sound is nearly always accompanied by a rapid crescendo coupled with a rapid rise in pitch and dynamics, and is generally very energetic in character (see Example 2).

(3) 'Middle Sound'. In light of Saunders' description of 'a process of fusion' between her two Elemental Sounds, I find it useful to identify and define a Middle Sound between them. This consists of sounds that are made up of harmonic trills on the lowest strings of the celli, violas and double bass (see Example $3 \mathrm{a}$ and $3 \mathrm{~b}$ ). While reminiscent of the second Elemental Sound's overtone-rich content, and using the harmonic trill, these sounds retain a muffled, indistinct quality, as they are performed on low strings.

(4) Shrill and metallic timbres. These have a piercing and direct quality ('metallic' meaning rich in strong high spectral components). These include most actions sul ponticello on the higher strings (often mixed with high string harmonics) and bowing behind the bridge, resulting in indefinite shrill high pitches. Additional metallic sounds result from the percussion, most markedly the spring coils and chains vibrating on the tam-tam and bass drum, tam-tam, metal plate and the metal grate.

(5) Fixed, stable pitch (or, more broadly, stationary sound). This is a conspicuous exception throughout Ire, standing out from its unstable surroundings. Even so, it is frequently blurred by the use of clusters, glissandi and/or fast changes in dynamics. I propose an analysis of its pitch structure below.

(6) Resonances. A sub-category of stationary sound, these sounds are quiet, long, uniform and often very low or high in register. Resonances are often masked by louder sounds, and are revealed as a result of their decay; the resonances themselves are not always decaying.

\footnotetext{
${ }^{6}$ Daniel Pressnitzer, Roy D. Patterson and Katrin Krumbholz, 'The Lower Limit of Melodic Pitch', The Journal of the Acoustical Society of America, 109, no. 5 (2001), pp. 2074-84. They suggest that the lowest melodic pitch - that is, a complex tone whose intervallic relationship to neighbouring tones is clearly recognisable to human ears - has a fundamental at around $30 \mathrm{~Hz}$ - approximately $\mathrm{B} 0$ - three semitones higher than the double bass's lowest note in Ire. The soloist's lowest notes on the detuned C string come very close to this limit too.
} 
Example 2.

Rebecca Saunders, Ire, solo cello part, bars 93-94. (C) Copyright 2016 Henry Litolff's Verlag.

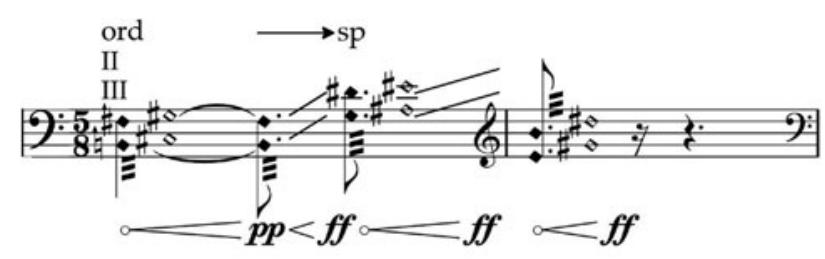

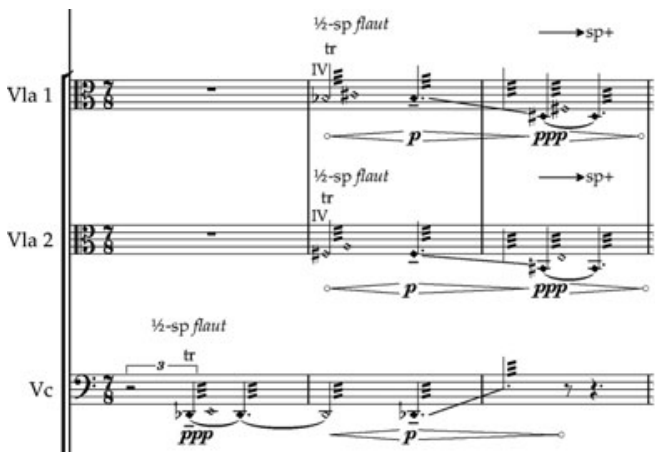

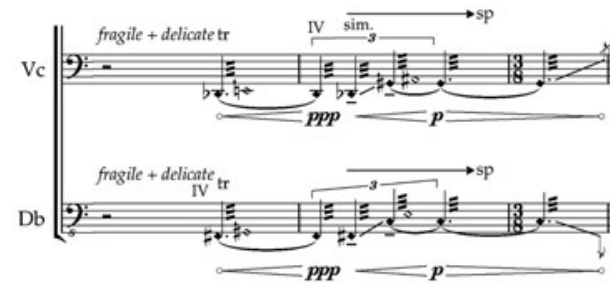

Example 3 .

(a) Rebecca Saunders, Ire, violas and ensemble cello parts, bars $27-29$. (C) Copyright 2016 Henry Litolff's Verlag. (b) Rebecca Saunders, Ire, ensemble cello and double-bass parts, bars 47-49. (C) Copyright 2016 Henry Litolff's Verlag.
(7) Bass sounds. These can be considered a separate category, with some overlap with Element 1 and Fixed pitch. These include continuous bowing on the double bass's fifth string, and some of the percussion sounds. While exact pitch is less salient in the extreme low range, instances of this category are more timbrally stable than Element 1 sounds, and are sometimes used in conjunction with pitched processes.

\section{Gestural and articulative elements}

It is possible to describe an additional group of elements, or gestures, which function as articulation within the timbral characteristics. Consequently, they can be inserted into various timbral categories and lend themselves to various combinations, making possible transitions and connections between sound categories.

(1) Trills and tremolos are omnipresent and central in both Elemental Sounds.

(2) Sharp attacks appear in various forms in the percussion part, but can also take the form of various string techniques including Bartók pizzicato, battuto, etc.

(3) Glissandi too are very common throughout. They appear frequently in both Elemental Sounds and are one of the main reasons behind the weakening of the perception of pitch in much of the piece.

In her interview, Saunders commented on the fusion of timbral characteristics and gestural elements which gives the two Elemental Sounds their identities:

The low lowered C string material is essentially gestural, exploring a very special timbral palette. The overtone-dominated loud material (cresc. to sul pont with up-bow of a double-trill of harmonics) is also a gestural cell, not just a timbral fragment, which creates a big timbral and expressive contrast to the low material. 


\section{Ire - phrase-based formal analysis}

Ire is formally structured as a chain of phrases of varying length. I will try to describe the logic behind this approach in a way that is grounded in the musical material; however, I readily admit that my hearing and intuition often played a central role in the analysis. Other listeners may legitimately dispute some of the segmentation I propose. My aural analysis was probably also informed by interpretive decisions made by the performing musicians. ${ }^{7}$

I propose the following cues for segmentation, which signal a new phrase:

- Silence;

- A significant feeling of repose in comparison to what came before and what begins after;

- One group of gestures coming to a conclusion and a new one beginning.

Generally speaking, each phrase can be identified through at least one primary feature: for example, a dominant sound category, a specific soloist-ensemble dynamic or a build-up to a local climax.

Some of these phrases may be broken down into sub-phrases between which some element of continuity is maintained. Often a continuous sound or resonance creates an overlap between one subphrase and the next, creating a larger process or momentum that prevents a feeling of conclusion. There is some overlap between my definitions of a phrase border and a sub-phrase border, my thesis being that, as the piece progresses, the phrases become generally longer, while phrase and sub-phrase borders become more difficult to tell apart. This coincides with the general dramatic trajectory of the whole piece, which gains energy and momentum as it progresses, with greater density and overlapping of events. Figure 1 shows an overview of my phrase division of Ire, based on primary segmentation cues: silences, contrasts and discontinuities.

Within these divisions into phrases, the introduction, varying dominance and interplay of various sound categories are important factors affecting the form of the piece as a whole. Table 1 provides a summarised overview of phrase content.

This overview suggests that each phrase contains between one and three dominant sound categories. The introduction of new sounds (or variations on earlier sounds, such as the first time Element 2 is played by the soloist) piques the listener's attention, contributing to the piece's overall momentum. Additionally, the subsequent rise in prominence and/or ubiquity of each new sound category stands out as a clear overall process threading throughout the piece. Alongside the rise in general energy and density of events, one can trace a clear meta-process in timbre, manifested in the distribution of the sound categories. The piece is initially dominated by low and mellow timbres; as it progresses, shrill and metallic timbres proliferate, eventually nearly completely drowning out the dark timbres. The reappearance of Element 1 towards the climax serves as a recapitulation of sorts.

Despite Saunders' description of a process of fusion between the two Elemental Sounds, it is difficult to find striking examples of

\footnotetext{
7 Rebecca Saunders, Ire, performed by Jean-Guihen Queyras (cello) and Ensemble Resonanz, conducted by Peter Rundel: www.youtube.com/watch?v=8HdKG2RYOyQ (accessed 26 January 2021).
} 
Figure 1 .

Rebecca Saunders, Ire: graphic representation of phrases (solid borders) and sub-phrases (dashed borders), with bar numbers, overlaid on summed mono waveform representation of the piece.

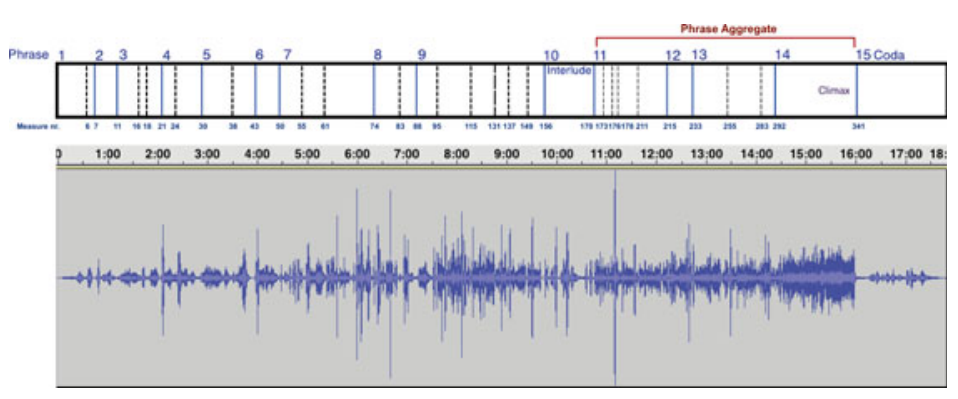

this. The two Elemental Sounds remain distinct from each other to the end. Although there are moments of ambiguity and overlap (as manifest in the Middle Sound), these do not at any point gain prominence and/or long duration. The final juxtaposition of Element 1 with the frenzied accumulation of sharp and energetic timbres in phrase 14 serves as a fusion only in the sense of overlaying the two simultaneously, rather than transforming either of them. Even so, the two groups of sounds occupy very distinct frequency ranges, somewhat facilitating the listener's differentiation of the two. Conversely, the low sounds are more easily masked by their loud surroundings, the threshold of hearing for such low frequencies being much higher.

Thus, the possibility of overlapping and morphological transformation across sound categories is rarely pursued, with Saunders opting instead for juxtapositions of contrasting textures. Perhaps it is the very rareness of these instances that intensifies their intriguing quality to my ears. In addition to the Middle Sound noted above, other examples include bar 47, where the bass drum is played with a superball, producing a dark timbre; at the same time the addition of a sizzle chain provides a hint of metallic timbres. Similarly, the piece's coda - phrase 15 is a fusion of the Resonances sound category (long and quiet sounds revealed after loud sounds) and the Shrill and metallic timbres category.

As mentioned before, the use of articulating elements across various sound categories (such as trills and tremolos) is important in giving the piece an overall sense of organic development and unity. It may be argued that the contrast between the two main sound categories is somewhat tempered by the presence of these common gestures, in spite of stronger contrasts in timbral qualities. The articulating elements also have an important function in giving the sound categories their identities. A good example occurs in phrase 5 , where the first clear combination of harmonic trills with shrill and metallic timbres reveals Element 2 for the first time as distinct from the more general category of Shrill and metallic timbres. The same can be said for the addition of trills, vibrato and other destabilising articulative elements to low pitches, thus differentiating between the Bass sounds category and Element 1.

When examining the phrase structure as a whole, it becomes clear that the piece is divided into two large parts. Each of these parts ends with a long, complex phrase (or aggregation of phrases) followed by a more restful phrase: phrases 1-9+ interlude (phrase 10), and phrases 11-14 + coda (phrase 15). Though phrase lengths change somewhat irregularly, they can be seen to grow longer overall, becoming less clearly outlined as the piece progresses. This is consistent with a logic of growing density and complexity of the material - with more processes and/or independent musical layers sustaining longer phrases and obstructing the clear delineation of the phrases' borders (one such independent process is pitched, as detailed below). The 
Table 1.

Overview of phrase sound contents in Ire

\begin{tabular}{|c|c|c|}
\hline Phrase & $\begin{array}{l}\text { Dominant categories/other } \\
\text { sounds }\end{array}$ & $\begin{array}{l}\text { New categories or specific sounds/notable internal } \\
\text { process }\end{array}$ \\
\hline 1 & Element 1 & Element 1 \\
\hline 2 & Element 1 & \\
\hline 3 & Element 1 & Resonance \\
\hline 4 & Element 1 & Middle Sound, transition to metallic timbres \\
\hline 5 & Element 1 & Element 2 \\
\hline 6 & Element 1 & \\
\hline 7 & $\begin{array}{l}\text { - Element } 1 \text { (Soloist) } \\
\text { - Pitch and Element } 2 \text { gain } \\
\text { prominence }\end{array}$ & Pitch, spring coils, strong attacks \\
\hline 8 & $\begin{array}{l}\text { - Pitch (first half), Element } 2 \\
\text { (second half) } \\
\text { - Strong attacks make a } \\
\text { conspicuous appearance }\end{array}$ & Element 2 in soloist \\
\hline 9 & $\begin{array}{l}\text { - Pitch stands out } \\
\text { - Element } 2 \text { more ubiquitous } \\
\text { and scattered } \\
\text { - Strong attacks become more } \\
\text { frequent }\end{array}$ & $\begin{array}{l}\text { - Pitch in soloist } \\
\text { - Short and dry timbres (bass-drum rim) }\end{array}$ \\
\hline $\begin{array}{l}10 \\
\text { (Interlude) }\end{array}$ & Element 1 & Sparse texture and contemplative atmosphere \\
\hline 11 & $\begin{array}{l}\text { - Element } 2 \\
\text { - Pitch }\end{array}$ & $\begin{array}{l}\text { Bass sounds (trace of Element 1); phrases } 11-14 \text { are } \\
\text { composed of many interweaving layers. Density of } \\
\text { events and energy level constantly increase towards the } \\
\text { end of phrase } 14 \text {. The reintroduction of Element } 1 \text { in } \\
\text { phrase } 14 \text { is an important formal marker }\end{array}$ \\
\hline 12 & $\begin{array}{l}\text { - Element } 2 \\
\text { - Pitch } \\
\text { - Sharp attacks }\end{array}$ & \\
\hline 13 & $\begin{array}{l}\text { - Element } 2 \\
\text { - Pitch } \\
\text { - Sharp attacks }\end{array}$ & \\
\hline 14 & $\begin{array}{l}\text { - Element } 1 \text { (Soloist) } \\
\text { - Element } 2 \text {, Metallic timbres, } \\
\text { sharp attacks (Ensemble) }\end{array}$ & \\
\hline 15 (Coda) & $\begin{array}{l}\text { - Shrill and metallic } \\
\text { - Element } 2 \text { (bass drum) } \\
\text { - Resonance }\end{array}$ & Extended resonance \\
\hline
\end{tabular}


early phrases (1-6) trace an arc in dynamics and/or range; they are, in a sense, self-contained, their endings not necessitating a continuation. Beginning with phrase 7 , an open end becomes the norm: nearly all subsequent phrases do not end in silence, a sense of conclusion becomes rare and phrases no longer finish in a clear descent in range or dynamics. This too is consistent with the described trajectory of the piece: with a sense of conclusion (or clear repose) becoming rarer, phrases and phrase aggregates tend to become longer. Phrases 11-14 are a culmination of this process, consisting of manifold layers, each with its own internal process and dominant sound category; my identification of their borders largely relies on moments where the endpoints of these layers overlap. Figure 2 presents an excerpt of my analysis of phrases $11-14$.

Further analysis suggested the following insights regarding phrase borders:

- Silence is the clearest method of unambiguously delineating phrases.

- Resonance is usually correlated with a sub-phrase border; however, in some instances a lack of a feeling of conclusion in some musical layers throws the existence of such a border into question. As the piece progresses, context can also throw new light on previous events, sometimes changing the perception of phrase and sub-phrase borders.

- Overlap (elision) of musical gestures makes phrase and sub-phrase borders ambiguous.

\section{Pitched processes in Ire}

As mentioned, stable pitch is a relatively rare and conspicuous case in Ire. Figure 3 shows a reduction of all instances of stable pitch. Solid bar lines indicate the division of pitched events into groups, which I identify as being related to each other. This relationship is based primarily on proximity but can also indicate some type of internal process. Dashed bar lines are used to indicate subdivisions within longer processes. Bar numbers are shown above the pitched events. Bass tones are shown only where I found that they may have a meaningful connection to the pitched processes. In describing the relation between pitch and timbre, we can differentiate between events where pitch is used chiefly in support of timbre and events where specific pitches are intentionally highlighted. Moreover, it appears to me that the oscillation between these two options is a central idea in the treatment of pitch in Ire.

Figure 3 reveals that pitch in Ire is almost exclusively in one of two configurations: the first consists of dense chords and/or clusters, often spread over a wide range (between one and two octaves), whereas the second consists of one pitch played by a number of instruments in unison (such as bar 103) or small microtonal clusters no larger than a halfstep, centred around one pitch (such as G in bar 88 and D in bar 192). Movement between these two configurations is usually achieved through collective glissandi.

To my ears, the chords and clusters are perceived mainly as timbre rather than pitch aggregates. Their width and density delineate a range of frequencies, while hindering the clear perception of any one pitch. As an exception to this rule, the extreme pitches of a chord or cluster may stand out, or the orchestration may cause some of a chord's components to be more noticeable. However, these chords are generally uniformly orchestrated, with all 

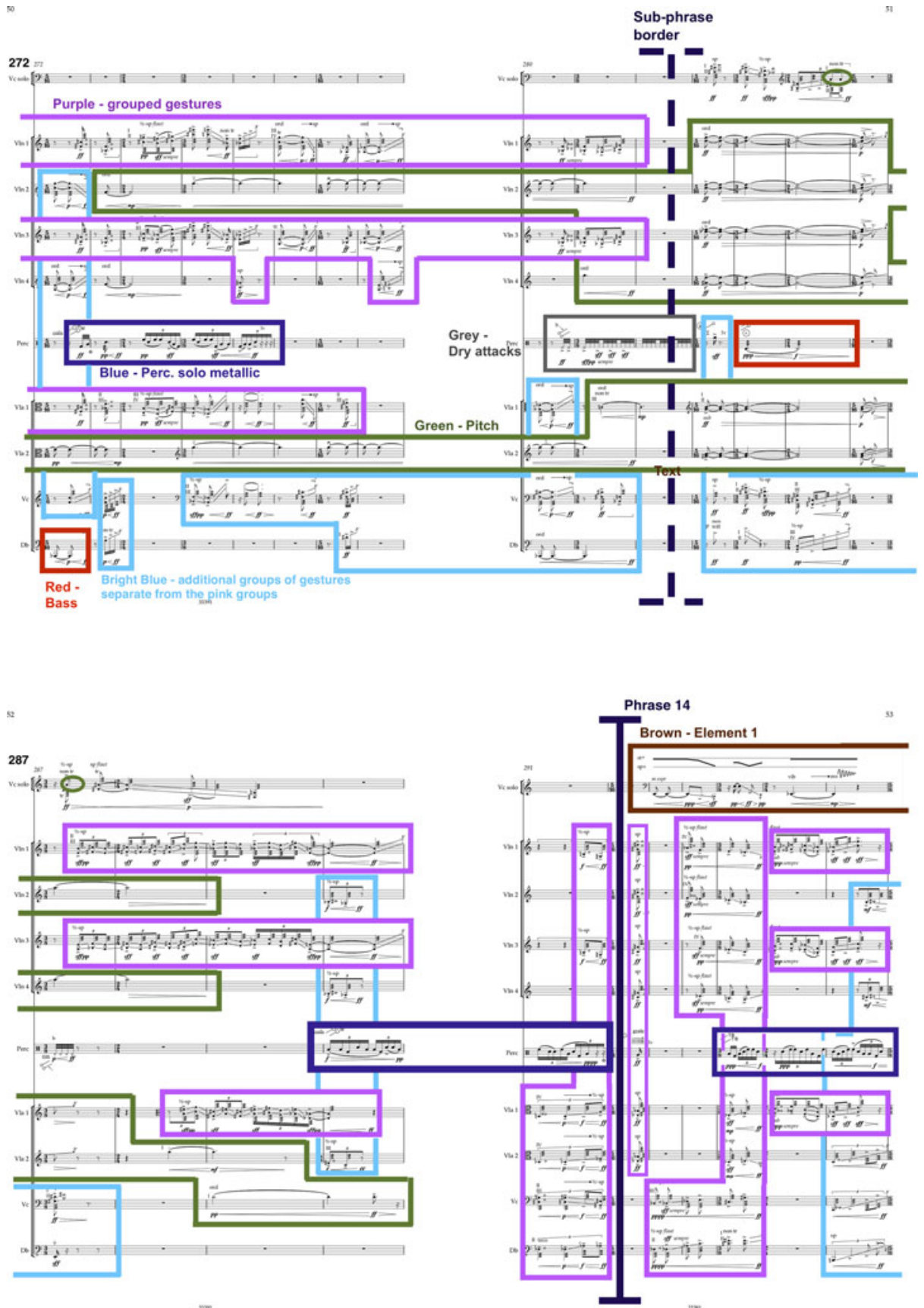

Figure 2

Partial analysis of layers in phrases 11-14. (C) Copyright 2016 Henry Litolff's Verlag. participating instruments assigned the same dynamics and similar playing techniques (bow position, low or high strings, harmonics). By contrast, doublings of a single pitch stand out. The same can be said for the small clusters, though in these cases the added microtones add 'fuzziness' to the perceived pitch.

In Figure 3, instances of clear pitch are indicated with a black arrow, and in these instances two main tendencies emerge. The first involves some pitches appearing more often than others, serving as local pitch 

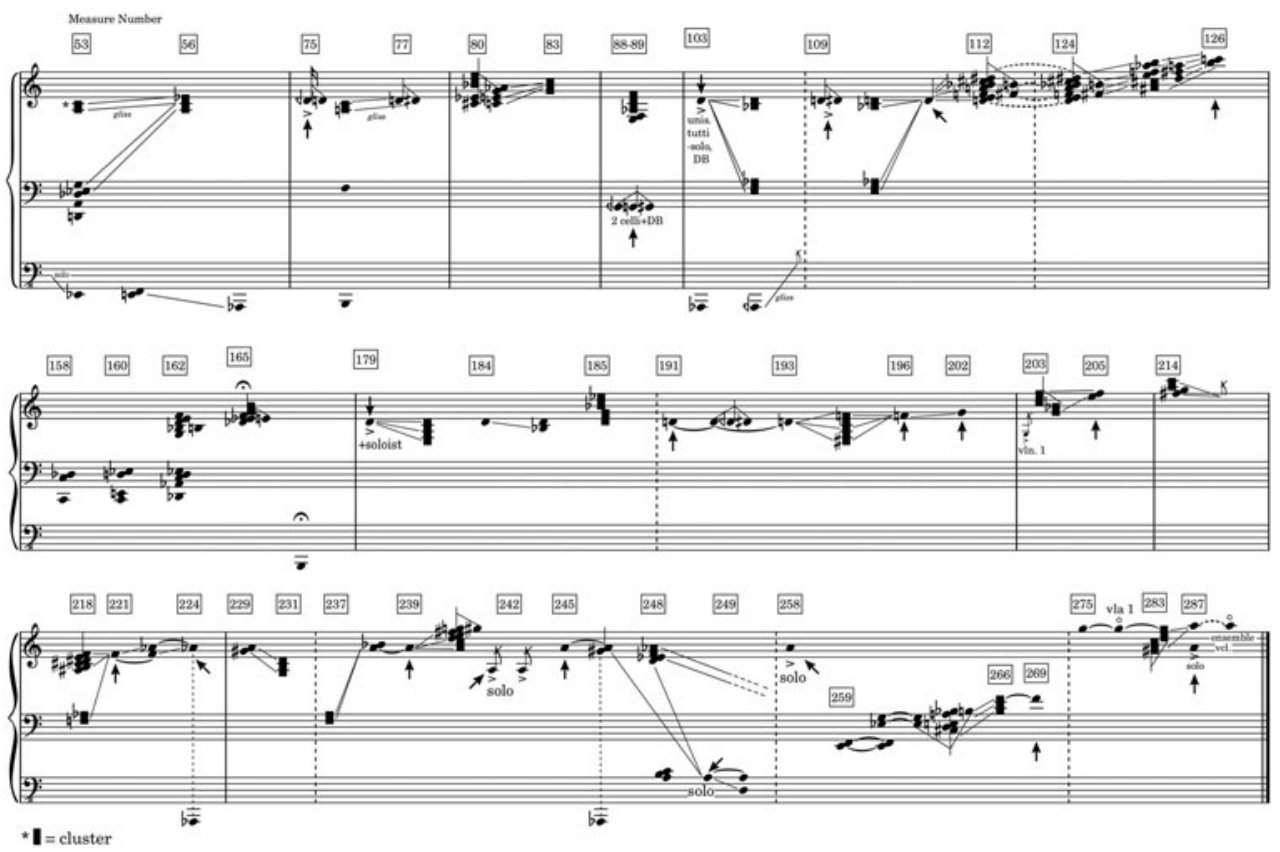

Figure 3.

Reduction of pitches in Ire. centres; the second consists of three upward pitched progressions, between bars 103 and 126, bars 179 and 205 and bars 221 and 287. The last progression ends on $\mathrm{A} 5$, one of the highest clear pitches in the piece. These pitch progressions are the closest one can find in the piece to a pitch-based formal device; the gradual movement between prominent pitches adds a unifying element to the piece's form, in a manner partially independent of the phrase structure. This is most true for the third progression, whose relative continuity (it is less fragmented than the previous progressions, its parts less far apart) and length, together with the fact that it leads to one of the piece's highest clear pitches, contributes to a sense of tension and build-up within phrases 12 to 13. The two pitches that appear more often than others $-\mathrm{D}$ and $\mathrm{A}-$ also contribute a sense of unity within the piece.

That progressions between clusters and clear pitches occur via glissandi suggests a sense of flux between these two states. The glissandi are akin to adjusting one's focus, moving between timbre and pitch perception. Thus, the potential ambiguity inherent in pitch between these two modes of perception is highlighted. This ambiguity of perception is especially apparent in regard to the lowest sounds. These tones are ubiquitous throughout the piece, mainly in the double bass part, whose lowest notes hover around the threshold of melodic pitch perception. While most of the bass sounds are treated in a timbral, gestural manner, there are passages in which bass notes have clearer relationships to the pitched processes. For example, in bar 103 , seven instruments playing an accented D4 in unison are answered a sixteenth-note later by the double bass's accented low A flat. This conjunction of accents pulls the bass note into sudden focus, showing its connection to the events above it and encouraging attention to its pitch. Similarly, in bar 167, the double bass plays a B0 immediately after a cluster whose highest note was B4.

The opposition between solo and tutti is also closely correlated with treatment of the pitch material. The majority of the pitched material 
appears in the ensemble, but not in the soloist's part. Instances where the soloist does contribute to the pitched category therefore stand out, further highlighting theses pitches. In bar 88 , for example, the soloist plays a $\mathrm{G}$ unison double-stop. This stands in stark contrast to the soloist's previous material and further highlights the clear pitch. In bar 179, the soloist and first violin, in the midst of a frenzied chain of gestures, join the second violin with an accented $\mathrm{D}$. This sudden and momentary breach in the separation between the different layers of events serves to throw additional focus on the pitch.

In summary, pitch has two main functions in Ire. In its first function, instances of clear and accented pitch are interwoven throughout the piece, giving it an additional level of formal cohesion. This observation is corroborated by Saunders:

Pitch [in Ire] occurs as a structural point of reference - as it is rarely audible then those moments of clear pitch stay logged in the listeners' memory and take on a heightened and quasi-structural significance.

Secondly, the use of pitch provides an opportunity to explore its complex relation to timbre. Pitch may be perceived as timbre in some cases, while becoming an aspect of timbre in others; this perceptive duality is key to the exploration in the work of sound materials as harbouring various potentialities.

\section{Still, for violin and orchestra}

Still is composed of two movements. I will focus on the first movement, which resembles the first 14 phrases of Ire in its process of accumulating energy and increasing density. The second, similar to Ire's coda (though much longer), is dominated by long and relatively quiet and delicate sounds.

\section{Sound materials}

The first movement of Still is dominated by a central gesture (see Example 4), explored by the solo violin, almost without stop, throughout the whole movement. This gesture is very similar to Element 2 in Ire. It consists of double harmonic trills on the violin's third and fourth strings, coupled with changing gradations of sul ponticello and rapid dynamic changes.

Apart from this gesture, the first movement contains a number of different sound materials:

(1) Pitch. Appearing mainly in the high strings and in the winds, stable pitch is a relatively common occurrence within the first movement. Similarly to Ire, it mainly takes one of two forms - wide clusters (bars 25-28 in the strings, 222-23 in the winds), and unison pitch doublings (bars 135-37 in the strings). As in Ire, small clusters are usually centred around one note: see, for example, bars 54-58 in the trombones, flutes and clarinets. Another similarity is the use of glissandi to rapidly transition between clusters and unisons. I have identified four such instances, all played by the string section and soloist: bars 127-28, 219, 164-65 and 224-25.

Pitches are often orchestrated with a constantly changing timbre. One typical example can be found in bars 37-44, where an E5F5 dyad migrates between an oboe, a clarinet and three trumpets with different mutes. 




Example 4.

Rebecca Saunders, Still, solo violin part, first movement, bars $1-8$. (C) Copyright 2016 Henry Litolffs Verlag.
(2) Very low or very high tones. As in Ire, Still makes extensive use of very low pitches, which are treated mainly as timbre and whose exact pitch content is of marginal importance. The orchestra contains three double basses with their fifth string tuned down to G0 (even lower than in Ire). Saunders comments in the piece's legend ${ }^{8}$ that in case the scordatura is too extreme, the string can be tuned slightly higher, as 'the unusual timbre is more important than the actual pitch'. Extreme low notes often appear in narrow clusters - as in bars 62 and 116. The bass trombone, tuba, bass clarinet and accordion contribute low clusters - for instance, in bars 87-88. Conversely, very high tones and clusters are often used, most often played by the accordion on the four-foot register; occasionally, similar sounds are produced by the violins bowing behind the bridge.

In her interview, Saunders mentioned her approach to the addition of extreme high or low tones to existing sounds, as a means by which to put a sound's timbre into a new perspective:

One can also add very very high or very very low sounds to a tone, thereby changing its 'frame' - that is, the space within which one hears the tone and the actual perceived timbre of said tone. This is comparable to taking a specific shade of red and by changing the canvas behind it from white to grey to black, etc., perceiving a completely different colour, shade or timbre. This is a form of orchestration of a sound.

(3) Imitations/Fragments of main gestures. Harmonic trills sul ponticello appear often in the violins, violas and celli. They are always much shorter than the soloist's gestures.

(4) Percussion and rhythmic impulses. The orchestra includes a large battery of percussion played by four musicians. These provide a wide palate of sounds that interact with or augment other sounds: shrill and metallic timbres (for example, tam-tam, spring coils), low indefinite pitch (for example, detuned timpani) and a variety of rhythmic impulses usually aligned with the soloist's actions. Rhythmic impulses can also be played by other instruments: most notably the piano, but also loud and short notes in the strings and winds.

(5) Resonance. Much as in Ire, soft and long sounds are often used to fill gaps between other sounds. Soft tremolos on the bass drum are common (as in bar 16); a variety of other sounds is also used, including clusters and low notes (that is, other categories can function as resonance). In a number of places, actual resonance - such as percussion resonance and the piano pedal - fills a pause.

\section{Phrasing}

In a manner perhaps more extreme than in Ire, the first movement of Still is made up of multiple short phrases that are almost exclusively

${ }^{8}$ Rebecca Saunders, Still (Leipzig: C. F. Peters, 2011). 
Table 2.

Overview of phrases in the first movement of Still

\begin{tabular}{|c|c|c|}
\hline $\begin{array}{l}\text { Phrase } \\
\text { aggregate }\end{array}$ & $\begin{array}{l}\text { Measure number } \\
\text { of phrase beginning }\end{array}$ & Sound types used \\
\hline \multirow[t]{7}{*}{1} & 1 & Central gesture $(\mathrm{C})$ \\
\hline & 3 & $\mathrm{C}$ \\
\hline & 6 & $\mathrm{C}$ \\
\hline & 10 & C, rhythmic impulse (Rt), low tone $(\mathbf{L})$ (resonance $(\mathbf{R s})$ ) \\
\hline & 13 & C, L, Rs \\
\hline & 17 & $\mathrm{C}$, harmonic trills (tr) (in orchestra), high tones $(\mathbf{H}), \mathrm{Rt}, \mathrm{Rs}$ (piano ped.) \\
\hline & 21 & C, L, pitch (Pt), percussion (Pr), Rs \\
\hline \multirow[t]{5}{*}{2} & 30 & $\mathrm{C}, \mathrm{L}, \mathrm{Pr}$ \\
\hline & 32 & Pt, C, Pr, tr, L, Rs \\
\hline & 46 & $\mathrm{C}, \mathrm{Rt}, \mathrm{L}$ \\
\hline & 49 & C, Pr, L \\
\hline & 52 & $\mathrm{C}, \mathrm{Pt}, \mathrm{Pr}, \mathrm{tr}, \mathrm{H}, \mathrm{L}$ \\
\hline \multirow[t]{4}{*}{3} & 70 & C, Rt, L \\
\hline & 77 & $\mathrm{Pt}, \mathrm{C}, \mathrm{L}, \mathrm{Rt}, \mathrm{Rs}$ \\
\hline & 84 & $\mathrm{C}, \mathrm{tr}, \mathrm{L}, \mathrm{Rt}, \mathrm{Pt}$ \\
\hline & 101 & $\mathrm{C}, \mathrm{L}, \mathrm{Pr}, \mathrm{Pt}, \mathrm{tr}, \mathrm{Rs}$ \\
\hline \multirow[t]{2}{*}{4} & 109 & $\mathrm{C}, \mathrm{Pt}, \mathrm{Rt}$ (percussion and brass), L, Rs \\
\hline & 124 & C, Pt, Rt, L, Rs \\
\hline 5 & 146 & $\mathrm{C}, \mathrm{Pt}, \mathrm{L}, \mathrm{tr}, \mathrm{H}, \mathrm{Rs}$ (low tones) \\
\hline \multirow[t]{4}{*}{6} & 154 & Pt, C, Rt, Pr, L \\
\hline & mid-163 & Pt, C, Rt, Pr, L, H, H \\
\hline & 178 & Pt, C, L, Pr, Rs \\
\hline & 184 & $\mathrm{Pt}, \mathrm{C}, \mathrm{L}, \mathrm{tr}, \mathrm{H}, \mathrm{Pr}$ \\
\hline \multirow[t]{6}{*}{7} & 199 & Pt, C, Pr, L, tr \\
\hline & 218 & $\mathrm{C}, \mathrm{Pt}$ \\
\hline & 221 & $\mathrm{C}, \mathrm{Pt}, \mathrm{Pr}$ (mainly metallic sounds), Rt \\
\hline & 232 & Pt, Pr-metallic, Rt (no central gesture) \\
\hline & 237 & Pt, Pr-metallic, Rt, H (accordion clusters) \\
\hline & 244 & Pt, Pr-metallic, Rt, $\mathrm{H}$ \\
\hline
\end{tabular}

centred around the soloist's actions. In my counting, there are 29 relatively short phrases divided by rests or moments of repose. Consequently, I find it useful to abandon the logic of large phrases and sub-phrases used in Ire, opting instead for multiple smaller phrases gathered in aggregates. As a starting point for the identification of the phrases' boundaries, I take moments where the soloist pauses. As was 


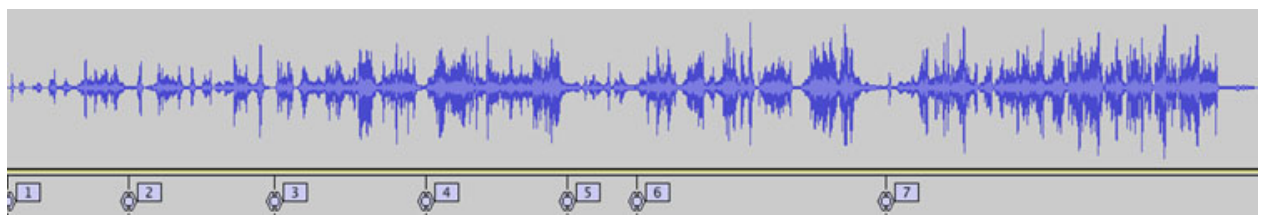

Figure 4 .

Waveform representation of first movement of Still, showing starting positions of phrase aggregates. with Ire's phrases, my division of phrase aggregates in Still is largely intuitive, based mainly on the length of the reposeful moments. Table 2 and Figure 4 summarise the first movement's phrases and delineate larger sections (aggregates) within the movement, including details about the sound types used within each phrase.

Interactions between elements in the first movement of Still

Unlike in Ire, one cannot trace a clear timbre-centred process within Still's first movement. Two sound types are in the foreground: the double harmonic trill central gesture, and pitched sounds (whether clusters or clear pitches). The only notable sound-type related event is the omission of the central gesture from the last three phrases. Pitch is foregrounded by the fact that it tends to appear uninterrupted for relatively long stretches (a few bars), while other sounds are used in a more haphazard and disconnected manner. Orchestration also plays a part in the foregrounding of pitch - it is usually played by large groups of instruments; the orchestration of changing timbre also serves to draw one's attention to the pitch. As in Ire, the stability of pitch stands in stark contrast to the unstable, restless and/or inharmonic attributes of most other sounds used.

In Still, the precise divisions between phrases seem to be both less ambiguous and less significant to the overall analysis than in Ire. Nevertheless, the comparison with Ire affords some insights. Phrases in Still are generally shorter and much more numerous. As in Ire, the phrase aggregates in Still tend to grow longer and their contents become denser as they progress. Another point of similarity is the insertion of one relatively short and calm phrase into this trajectory: phrase 10 in Ire and phrase aggregate 5 in Still.

With no clear process in the timbral realm, does a different formal idea or motivation underpin the organisation of sounds within Still's first movement? In Saunders' foreword to the score, she writes:

Fragments, each time slightly varied, gradually create a single image... like a giant mobile seen from many perspectives, that in itself remains untouched.

And in her interview, she says:

It is increasingly important for me nowadays to explore static harmonic fields, but this always from the standpoint of timbre and the kind of specific sound material I am working with. A melodic line or a harmonic progression imply a goal-orientated progression, but I wish more to work within static fields of timbre and their inherent harmonies - creating multiple perspectives of a single sonic landscape.

These words can throw light on the movement's construction. The solo violin's central gesture is reiterated constantly, often in short bursts, while varying sounds are added to it, showing it, in Saunders' words, from many perspectives, within a relatively static timbral field dominated by the central gesture. Saunders goes on to say that: 
One can also change the sounds/silences/resonances before and after a given tone - the context will also sharply re-define how the same said tone is perceived. In this respect no sound is 'new' and no timbre is 'absolute' but always defined by its context.

This too throws light on both Still and Ire. The music's many pauses (with or without resonance) serve the compositional goal of re-contextualising sounds in ever new ways.

\section{Conclusions}

My analyses of Rebecca Saunders' Ire and Still demonstrate the centrality of timbre to their compositional logic. The sounds used in the pieces can be grouped into clearly delineated timbre-based categories, which afford various interactions and gradations. Moreover, a sonic environment rich in sounds that are inharmonic, in constant motion and/or occupying extreme ranges of human hearing serves to highlight and foreground 'ordinary', stable pitch in a way that can be useful for such pieces' formal cohesion, as is most evident in Ire. In the first movements of both Ire and Still similar devices contribute to the perception of growing energy and density. Phrases (or phrase aggregates) grow generally longer, and the density of events grows, though in Still the use of overlapping layers is not as complex and central as in Ire. Still's first movement also lacks the overarching timbrebased formal process found in Ire. Two sound types - the soloist's central gesture and pitch - are foregrounded and juxtaposed throughout the whole movement, while other sound types are used in an articulative and additive fashion.

Moments of repose, in their many forms and gradations, are a central aspect of both pieces' compositional rhythm. In Ire, these moments serve as punctuation points in the piece's overall form; in Still, they function more as facilitating many iterations of the central gesture, in the attempt to reveal it from 'different angles'. This demonstrates a central idea in Saunders' composition process - in her words, providing 'multiple perspectives' of a single sound. This is achieved via timbre manipulation and re-contextualisation - the placement of the same sound within various sonic environments. I find this idea useful in augmenting the pieces' analysis, especially at the micro level - inviting scrutiny of the many ways in which various sounds are joined, and the ways in which this affects one's perception.

For me, perceptual ambiguity as a compositional tool for encouraging multiple possible perceptual groupings and interpretations of the same sounds has proven to be the most intriguing concept arising from the analysis of Ire. Timbre is an extremely rich sonic domain, allowing great flexibility and many gradations. Coupled with differing contexts and/or layered with differing articulations, timbre can enable changing perceptions of the groupings and connections of various sounds, allowing for exploration of the borderline between perception of pitch and timbre. Finally, timbre can function at the level of macroform, through the multidimensional interaction between textural layers and phrase divisions. I hope to have demonstrated that timbrecentred analysis is an approach that could be used to examine other music, both by Saunders and many other composers. 